Documentation et bibliothèques

DOCUMENTATION BIBLIOTHËUES

\title{
La constitution des collections
}

Collection Development

El establecimiento de las colecciones

\section{Maryse Trudeau, Isabelle Charuest et Michèle Lefebvre}

Volume 51, numéro 1, janvier-mars 2005

Bibliothèque nationale du Québec

URI : https://id.erudit.org/iderudit/1030123ar

DOI : https://doi.org/10.7202/1030123ar

Aller au sommaire du numéro

Éditeur(s)

Association pour l'avancement des sciences et des techniques de la documentation (ASTED)

ISSN

0315-2340 (imprimé)

2291-8949 (numérique)

Découvrir la revue

Citer cet article

Trudeau, M., Charuest, I. \& Lefebvre, M. (2005). La constitution des collections. Documentation et bibliothèques, 51(1), 51-53. https://doi.org/10.7202/1030123ar

\section{Résumé de l'article}

Le développement de la collection universelle de prêt et de référence repose sur l'application d'une politique générale de développement de la collection, complétée par des lignes directrices, des portraits et des profils de collection. Un vaste programme d'acquisition a permis d'enrichir les collections initiales, en combinant les efforts de bibliothécaires sélectionneurs, des libraires agréés du Québec et le recours à des consortiums d'acquisition.
Tous droits réservés (c) Association pour l'avancement des sciences et des techniques de la documentation (ASTED), 2005
Ce document est protégé par la loi sur le droit d'auteur. L'utilisation des services d'Érudit (y compris la reproduction) est assujettie à sa politique d'utilisation que vous pouvez consulter en ligne.

https://apropos.erudit.org/fr/usagers/politique-dutilisation/ 


\section{La constitution des collections}

\section{MARYSE TRUDEAU}

Directrice des acquisitions et du traitement documentaire de la collection de prêt et de référence

\section{ISABELLE CHARUEST \\ Coordonnatrice}

Section des acquisitions et du traitement documentaire de la collection de prêt et de référence

\section{MICHÈLE LEFEBVRE \\ Bibliothécaire}

Direction des services aux milieux

documentaires

\section{RÉSUMÉ | ABSTRACTS | RESUMEN}

Le développement de la collection universelle de prêt et de référence repose sur l'application d'une politique générale de développement de la collection, complétée par des lignes directrices, des portraits et des profils de collection. Un vaste programme d'acquisition a permis d'enrichir les collections initiales, en combinant les efforts de bibliothécaires sélectionneurs, des libraires agréés du Québec et le recours à des consortiums d'acquisition.

\section{Collection Development}

The development of a circulating and reference collection depends on the application of a general collections development policy, complemented by general guidelines and collection profiles. A vast acquisition programme is supported by the combined efforts of collections librarians, accredited bookstores and acquisitions consortia and has greatly enriched the core collections.

\section{El establecimiento de las colecciones}

El desarrollo de la colección universal de préstamo y consulta se basa en la aplicación de una política general de desarrollo de la colección, completada por ciertas directrices, retratos y perfiles de colección. Un vasto programa de adquisición permitió enriquecer las colecciones iniciales, combinando los esfuerzos de los bibliotecarios encargados de la selección, libreros acreditados de Quebec y el uso de consorcios de adquisición.
A lORS QUE LA COllection NATIONALE vise l'exhaustivité et s'enrichit principalement par le dépôt légal, le développement de la collection de prêt et de référence implique sélection et élagage. Pour répondre aux besoins de ses diverses clientèles, la BNQ vise à constituer un fonds documentaire de grande qualité, bien équilibré et régulièrement tenu à jour, pluraliste, pour refléter les besoins variés, et accessible gratuitement dans les meilleurs délais. Ce sont là les grands objectifs de la Politique générale de développement de la collection de prêt et de référence adoptée par la BNQ.

Au quotidien, ce développement est assuré par une équipe de bibliothécaires professionnels expérimentés et s'appuie sur des outils que la BNQ rend disponibles aux bibliothèques sur son portail Internet. Ces outils sont essentiellement au nombre de trois :

- Les lignes directrices d'une collection régissent la sélection, l'acquisition et la conservation de collections générales touchant plusieurs secteurs de la Bibliothèque, par exemple le développement de la collection de revues et journaux ou encore la collection de référence;

- Le portrait de collection présente les grandes orientations d'une collection particulière: composition, répartition interne, clientèle spécifique à laquelle elle s'adresse, objectifs de développement et critères généraux de sélection. Des portraits de collections ont été établis, entre autres, pour la collection Jeunesse et les collections de films;

- Le profil de collection s'applique, pour sa part, à une collection particulière, comme les sciences et la technologie. Il permet de consigner toutes les informations fines se rapportant à la sélection des documents. On y détermine les objectifs d'utilisation, les langues à développer, les sources de sélection, les consignes générales, les exclusions particulières, etc.

Pour chaque sujet et chaque type de document, on précise les critères spécifiques, les niveaux de difficulté de lecture souhaités, le nombre d'années de publication que l'on veut couvrir en acquisition rétrospective et le niveau d'exhaustivité recherché. 
Trepanier, Peter, De causis et tractatibus XLIII: [stone book], Hull, Galerie Axe Néo-7 art contemporain et l'artiste, 1994. Livre d'artiste, $34 \times 26,5 \mathrm{~cm}$.

À partir de ces objectifs et de cadres normatifs, des collections ont été constituées, réunissant des ressources existantes et de nouvelles acquisitions. N'ayant, à toutes fins pratiques, jamais fait l'objet d'élagage, les fonds de la Bibliothèque centrale de Montréal ont été examinés pour en éliminer les documents détériorés et les remplacer, pour retirer les éditions ou exemplaires addi-

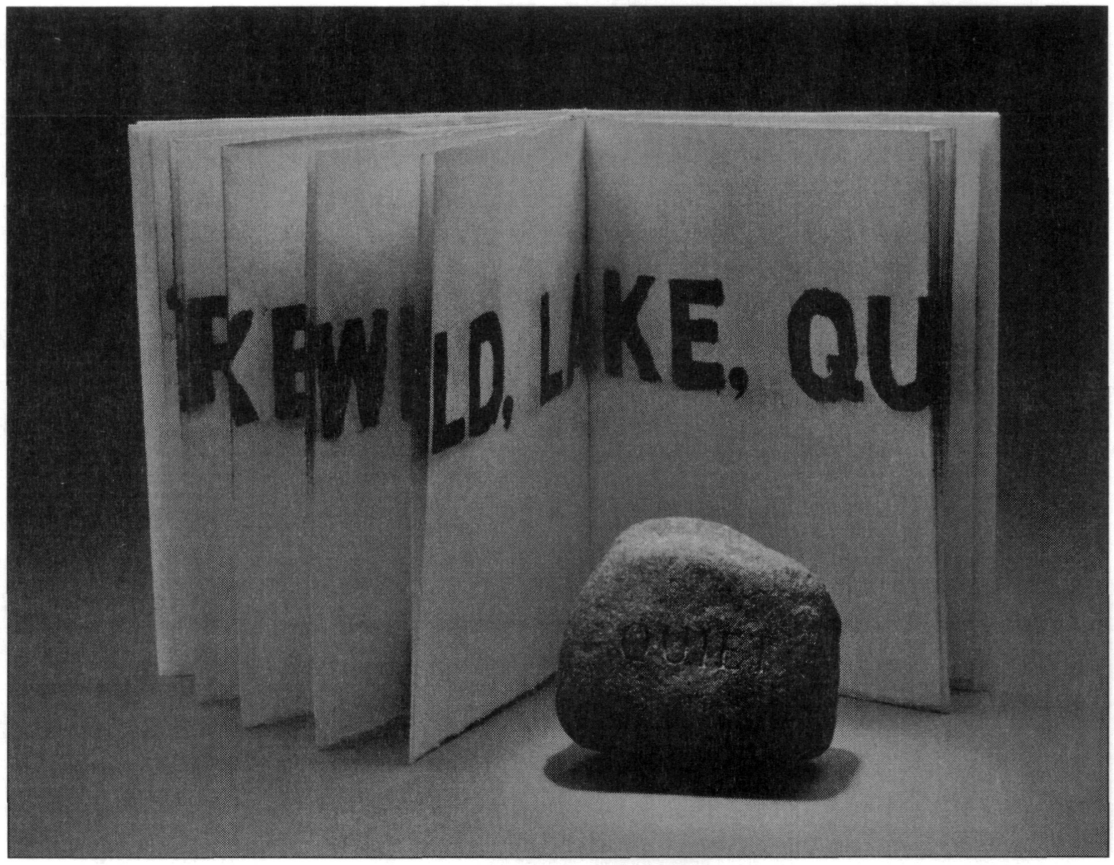
tionnels non requis, ou encore, dans certaines disciplines comme l'informatique ou les sciences exactes, pour retirer les titres périmés ne présentant pas d'intérêt historique ou patrimonial. La Collection nationale s'est enrichie de quelques milliers de documents québécois présents à la $\mathrm{BCM}$ mais encore absents dans la collection nationale de diffusion, parfois également absents dans la collection de conservation.

\section{DE NOUVELLES ACQUISITIONS}

Depuis l'automne 2001, l'équipe de la Section des acquisitions et du développement de la collection de prêt et de référence s'affaire à enrichir cette collection de nouveautés et de titres parus antérieurement. Pour la sélection des 475000 documents visés, cette équipe travaille en collaboration étroite avec les bibliothécaires de la Direction des services à la clientèle et compte sur la contribution externe de plusieurs professionnels d'expérience provenant de diverses régions du Québec. La démarche touche tous les types de documents: livres, ouvrages de référence, revues et journaux, enregistrements sonores et vidéo, livres lus, partitions musicales, cartes et plans, sans oublier les ressources électroniques, aujourd'hui incontournables: bases de données et autres ressources d'information électroniques, logiciels, livres électroniques, etc. Chacune de ces composantes a exigé une démarche spécifique qui devait tenir compte des sujets et des supports couverts, de la durée de vie utile des documents et de l'existence ou non d'une collection développée par la BCM. Pour chaque domaine de connaissance, il a fallu déterminer si, en plus des nouveautés, il était pertinent d'acquérir des ouvrages plus anciens. Dans la plupart des cas, il a été jugé utile de recourir à cet enrichissement rétrospectif, de façon ciblée.

Tout au long du processus, la BNQ s'est assurée de couvrir l'édition québécoise, canadienne et étrangère, en donnant évidemment priorité aux deux premières. Certains sujets ont demandé des efforts particuliers, soit parce que les ouvrages accessibles au grand public sont plus rares dans ces domaines (par exemple les sciences pures), soit parce que la Grande Bibliothèque s'est engagée à poursuivre un développement de pointe dans ce secteur, comme c'est le cas pour la collection Économie et affaires.

La BNQ a pu compter sur la collaboration efficace d'une centaine de libraires québécois agréés pour l'assister dans cette opération d'envergure d'acquisition de titres récents et plus anciens, pas toujours disponibles au Québec.

Cette réflexion s'est poursuivie au-delà du document imprimé, pour les enregistrements sonores et vidéo, les logiciels, les documents électroniques, etc. Dans ces domaines, il a fallu définir les supports privilégiés par la $\mathrm{BNQ}$ et apprécier le dédoublement nécessaire entre chacun d'eux. Ainsi, doit-on acquérir un film sur support DVD, sur vidéocassette ou sur les deux? Doit-on privilégier le support imprimé ou électronique dans le choix d'une ressource de référence? Internet est-il préférable au cédérom dans le cas des bases de données? Autant de questions auxquelles il a été donné des réponses nuancées, afin de permettre une adaptation continue à la réalité.

\section{DE NOUVELLES COLLECTIONS}

En plus des collections existantes, revues et augmentées, la BNQ propose aux usagers de la Grande Bibliothèque plusieurs collections totalement nouvelles: 
- une collection pour les jeunes, comprenant 70 ooo documents sur différents supports, en français, en anglais et dans quelques-unes des langues des nouveaux arrivants et des communautés culturelles;

- une collection en économie et affaires;

- une collection pour les nouveaux arrivants;

- une collection de films : des nouveautés, des films de répertoire et des documentaires;

- une collection de cartes et plans: des cartes géographiques, topographiques, maritimes, des cartes pour les randonnées pédestres, etc.;

- une collection multilingue, réunissant au départ 20000 documents, et des documents électroniques dans une dizaine de langues des nouveaux arrivants et des communautés culturelles présentes dans la population desservie.

Lors du lancement du nouveau portail de la BNQ, en janvier 2005 , la collection numérique de diffusion proposait déjà des bases de données électroniques, des images numériques, des enregistrements sonores en format numérique et des livres électroniques, de même qu'une liste imposante de revues et journaux, d'ici et d'ailleurs, accessibles à distance. Les ressources électroniques sont également très présentes dans la collection de référence: dictionnaires en ligne, encyclopédies, annuaires, répertoires statistiques, index de périodiques généraux et spécialisés, index de l'actualité. Il suffira de mentionner des titres tels: $L a$ Presse, Le Devoir, le Financial Times, Encyclopaedia Universalis, l'Annuaire du Canada, Grove Music Online pour donner un aperçu de la diversité des ressources électroniques disponibles.

Parallèlement à ces outils de référence traditionnels, la BNQ offre également des sources spécialisées originales et personnalisées, tels un répertoire d'études de marché, une base d'aide au choix de carrière, etc.

La BNQ effectue ces achats individuellement ou en commun, notamment par le biais du Consortium d'acquisition de ressources électroniques du Québec (CAREQ) ou celui de la Conférence des recteurs et des principaux des universités du Québec (CREPUQ). Né de l'initiative de la BNQ en 2003, le CAREQ négocie l'accès à des bases de données et des ressources électroniques à large diffusion pour l'ensemble des bibliothèques publiques du Québec et leurs abonnés. En août 2004, le CAREQ a ainsi conclu sa première entente avec Encyclopaedia Universalis, permettant aux usagers de la BNQ ainsi qu'à ceux des 39 bibliothèques autonomes participantes et du Réseau Biblio du Québec de consulter cette ressource par Internet, dans les bibliothèques ou à distance: plus de 3 millions de Québécois ont maintenant accès à la version électronique enrichie de cette encyclopédie fort utile pour les recherches personnelles ou scolaires. $\odot$

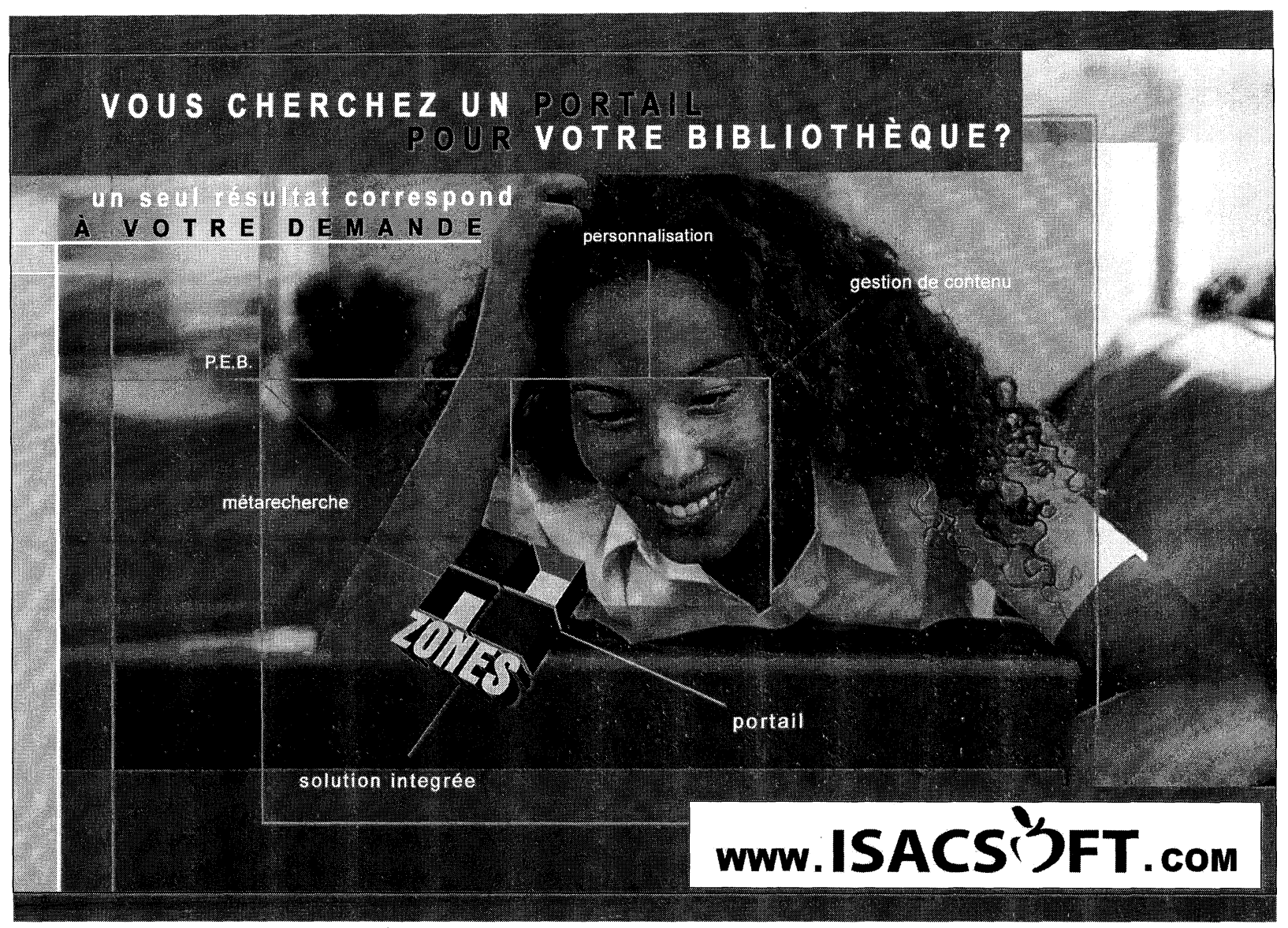

